\title{
OPEN A retrospective study assessing the acceleration effect of type I Helicobacter pylori infection on the progress of atrophic gastritis
}

\author{
Weidong Liu ${ }^{1,3}$, Junjie Tian $^{2,3}$, Wenjia Hui ${ }^{1}$, Wenjie Kong ${ }^{1}$, Yan Feng ${ }^{1}$, Junqiang $\mathrm{Si}^{2 \bowtie} \&$ \\ Feng Gao ${ }^{1 \bowtie}$
}

Based on the antibody typing classification, Helicobacter pylori infection can be divided into type I $H$. pylori infection and type II $H$. pylori infection. To observe the effects of different $H$. pylori infection types on the distribution of histopathological characteristics and the levels of three items of serum gastric function (PG I, PG II, G-17). 1175 cases from October 2018 to February 2020 were collected with ratio 1:2. All patients were performed with ${ }^{14} \mathrm{C}$-Urea breath test $\left({ }^{14} \mathrm{C}-\mathrm{UBT}\right), \mathrm{H}$. pylori antibody typing classification, three items of serum gastric function detection, painless gastroscopy, pathological examination, etc. According to $\mathrm{H}$. pylori antibody typing classification, patients were divided into three groups: type I H. pylori infection group, type II H. pylori infection group and control group. Significant difference existed among type I $H$. pylori infection group, type II $H$. pylori infection group and control group in inflammation and activity $\left(\chi^{2}=165.43,354.88, P\right.$ all $\left.<0.01\right)$. The proportion of three groups in OLGA staging had statistic difference $\left(\chi^{2}=67.99, P\right.$ all $\left.<0.01\right)$; Compared with type II $H$. pylori infection group and control group, the level of pepsinogen I, pepsinogen II, gastrin17 in type I H. pylori infection group increased, and PG I/PG II ratio (PG I/PG II ratio, PGR) decreased, which was statistically significant $\left(\chi^{2}=35.08,166.24,134.21,141.19 ; P\right.$ all $\left.<0.01\right)$. Type I $H$. pylori infection worsened the severity of gastric mucosal inflammation and activity. H. pylori infection was prone to induce atrophy of gastric mucosa, while type I $H$. pylori infection played a key role in promoting the progress of atrophic gastritis and affected the level of serum gastric function. The study indicated that the eradication of $\boldsymbol{H}$. pylori should be treated individually.

Helicobacter pylori (H. pylori), a single polar, multiple flagellum and spirally curved gram-negative bacilli belongs to the micro aerobic bacteria, whose main infection site is the stomach and duodenal sphere. H. pylori has a significant correlation with chronic gastritis with gastric mucosal atrophy and erosion, peptic ulcer, MALT lymphoma and gastric cancer (GC) $)^{1,2}$. About $20 \%$ of $H$. pylori infected patients develop precancerous lesions but only $2 \%$ develop gastric cancer, which is closely related to the host genetic, environment and virulent strains of $H$. pylori $i^{3}$. H. pylori has different characteristics of virulence of the infecting strain. The pathogenesis of $H$. pylori may be associated with a variety of pathogenic factors, including cytotoxin associated protein A (cytotoxin associated protein $\mathrm{A}, \mathrm{Cag} \mathrm{A}$ ), vacuolating cytotoxin $\mathrm{A}$ (vacuolating cytotoxin $\mathrm{A}$, VacA), duodenal ulcer promoting gene $\mathrm{A}$ and Adhesin, etc ${ }^{4,5}$. CagA and VacA are the two important determinants of $H$. pylori toxicity, inducing the damage of epithelial cells and chronic inflammation, which may ultimately lead to $\mathrm{GC}^{6}$. Based on the expression of serum virulence protein, the type of $H$. pylori infection is determined by antibody typing classification. If express CagA or VacA, it can be diagnosed as type I H. pylori infection, if not express CagA or VacA but Urease, it can be diagnosed as type II $H$. pylori infection.

Atrophic gastritis (AG) mainly caused by bad eating habits, smoking, drinking and H. pylori infection ${ }^{7}$. which is characterized by the decrease or disappearance of gastric acid glands, or accompanied by pylorus gland metaplasia and intestinal metaplasia (IM). A large sample cohort study has shown that $H$. pylori positive patients experienced chronic gastritis to AG and eventually to intestinal metaplasia, and the risk of gastric cancer is also

${ }^{1}$ Department of Gastroenterology, People's Hospital of Xinjiang Uygur Autonomous Region, No. 91 Tianchi Road, Tianshan District, Urumqi 83000, China. 'Department of Physiology, Shihezi University of Medicine, Shihezi, China. ${ }^{3}$ These authors contributed equally: Weidong Liu and Junjie Tian. ${ }^{\boxplus}$ email: sijunqiang@shzu.edu.cn; xjgf@ sina.com 
at a staircase increase ${ }^{8}$. Serological tests, including gastrin 17 (G-17) and pepsinogen (PG), could help to identify individuals at risk for AG. PG is an inactive precursor of pepsin in gastric juice and a special biological marker of gastric secretion. According to immunological activity and biochemical characteristics, PG can be divided into two categories: PGI and PGII, the former is mainly secreted by the chief cell and mucous neck cells, while the latter is secreted by fundus gland, cardiac glands, pyloric glands and Brunner's glands. Most of the synthetic PG is released into the gastric cavity, and only about $1 \%$ enter the blood circulation ${ }^{9}$. In ideal state, serum PG can accurately reflect the state of gastric mucosal lesion ${ }^{10-12}$. G-17 is an important gastrointestinal peptide hormone and produced in the antrum by G cells, which is correlated with the position and degree of gastric mucosal atrophy. When the synthesis of G-17 decreases, it could indicate the presence of AG in the antrum, while the presence of AG in the corpus can led to the increase of G-17 by the negative feedback of gastrin gastric acid axis ${ }^{13}$. This study aims to analyze the relationship between different types of $H$. pylori infection on the distribution of histopathological characteristics and the levels of three items of serum gastric function. As well as to provide a theoretical basis for the clinical individual eradication of $H$. pylori and a possible mechanism of type I $H$. pylori infection in the development of AG.

\section{Methods}

Subject investigated. In this retrospective analysis, 1175 inpatients were recruited in department of gastroenterology of people's hospital of Xinjiang Uygur Autonomous Region from October 2018 to February 2020. All inpatients were performed with ${ }^{14} \mathrm{C}$-Urea breath test $\left({ }^{14} \mathrm{C}\right.$-Urea breath test, $\left.{ }^{14} \mathrm{C}-\mathrm{UBT}\right), \mathrm{H}$. pylori antibody typing classification, three items of serum gastric function detection (PGI, PGII, G-17), painless gastroscopy, pathological examination, etc. Exclusion criteria: ethnic minorities; History of previous gastric cancer surgery; Result of ${ }^{14} \mathrm{C}$-UBT and $H$. pylori antibody typing classification was not conformed. Based on $H$. pylori antibody typing classification, all inpatients were divided into three groups: type I $H$. pylori infection group, type II $H$. pylori infection group and control group. 235 inpatients were enrolled in type II $H$. pylori infection group and the other two groups were 470 inpatients respectively (1:2 paired). Written and informed consent was obtained from each participant older than 18 years old. The results of the test will be presented anonymously, which complied with the principles of the Declaration of Helsinki. This study also approved by the ethics committee of people's hospital of Xinjiang Uygur Autonomous Region (KY2019051528).

${ }^{14}$ C-UBT. Inpatient took a ${ }^{14} \mathrm{C}$ Urea capsule $(0.75 \mu \mathrm{ci})$ on an empty stomach and sat quietly for $25 \mathrm{~min}$, then blew bubbles to the gas collecting bottle until the color of the liquid indicator changed from pink to colorless. Added $4.5 \mathrm{ml}$ scintillation solution to the gas collecting bottle, reversed three times and put into the H. pylori detector (HUBT-01A, headway) then the detection time set at $1 \mathrm{~min}$. The detection value $\geq 100 \mathrm{dpm}$ was identified to be $H$. pylori positive and the value $<100 \mathrm{dpm}$ was identified to be negative.

H. pylori antibody typing classification. Fasting venous blood $2-3 \mathrm{ml}$ with a procoagulant tube was collected and separated. H. pylori antibody typing classification assay kit (western blotting) was provided by Shenzhen bolt biotech Co., Ltd. Experimental process through serum antibody combined with dot blot membrane, enzyme-linked reaction, chromogenic reaction and terminal reaction, and the result determined by the standard band. According to $H$. pylori antibody typing classification, $H$. pylori infection was divided into type I $H$. pylori infection group, type II $H$. pylori infection group and control group. If expressed CagA or VacA, it could be diagnosed as type I $H$. pylori infection. If not expressed CagA and VacA but Urease A or Urease B, it could be diagnosed as type II $H$. pylori infection. If only expressed the quality control, which could be diagnosed as $H$. pylori negative. The appearance of band of quality control did not indicate that the test was invalid.

Three items of serum gastric function detection. Fasting venous blood 2-3 ml was collected by vacuum tube, then the serum was separated. PGI, PGII, G-17 test kit (Fluorescence immunochromatographic assay) was provided by BIOHIT Healthcare (Hefei) Co., Ltd. Adding $80 \mu \mathrm{l}$ serum to the test kit and placing in the Fluorescence immunoquantitative analyzer (HIT-91A) 15 min later. The results could be read by fast detection. The normal reference range of PGI, PGII, PGR and G-17 was 70-165 $\mu \mathrm{g} / \mathrm{l}, 3-11 \mu \mathrm{g} / \mathrm{l}, 7-20$ and 1-7 pmol/l, respectively.

Gastroscopy and pathological diagnosis. The diagnostic criteria for gastroscopy and pathology were based on Chinese consensus on chronic gastritis $(2017 \text {, Shanghai })^{14}$. Five gastric mucosa biopsies were taken according to the standard, including two gastric antrum biopsies, two gastric body biopsies and one gastric corner biopsy. Histopathological evaluation was performed according to the Sydney system. Chronic inflammation, activity, atrophy and intestinal metaplasia were graded as absent (0); mild (1); moderate (2) and severe (3).

Statistical analysis. SPSS17.0 software was used to analyze the data. Continuous variables were described as mean ( \pm standard deviationand), while categorical variables were described as percentages or frequencies, while categorical variables were described as percentages or frequencies. All data were tested for normal distribution by Shapiro-Wilk test. Kruskal-Wallis test was used to compare multiple quantitative variables, while chi-square test or Mann-Whitney $U$ were used for pairwise comparison between groups. $P$ value less than 0.05 was considered statistically significant. 


\begin{tabular}{|c|c|c|c|c|c|}
\hline Group & Type I H.pylori infection group & Type II H. pylori infection group & Control group & & \\
\hline$n$ & 470 & 235 & 470 & $\chi^{2}$ & $P$ value \\
\hline \multicolumn{6}{|c|}{ Inflammation } \\
\hline 0 & $9(1.9 \%)$ & $5(2.2 \%)$ & $25(5.3 \%)$ & \multirow{4}{*}{350.67} & $<0.01$ \\
\hline 1 & $139(29.6 \%$ & $162(68.9 \%)$ & $394(83.8 \%)$ & & $<0.01$ \\
\hline 2 & $306(65.1 \%)$ & $68(28.9 \%)$ & $51(10.9 \%)$ & & $<0.01$ \\
\hline 3 & $16(3.4 \%)$ & $0(0 \%)$ & $0(0 \%)$ & & $<0.01$ \\
\hline \multicolumn{6}{|l|}{ Activity } \\
\hline 0 & $153(32.5 \%)$ & $178(75.7 \%)$ & $421(89.6 \%)$ & \multirow{4}{*}{354.88} & $<0.01$ \\
\hline 1 & $217(46.2 \%)$ & $45(19.2 \%)$ & $41(8.7 \%)$ & & $<0.01$ \\
\hline 2 & $95(20.2 \%)$ & $12(5.1 \%)$ & $8(1.7 \%)$ & & $<0.01$ \\
\hline 3 & $5(1.1 \%)$ & $0(0 \%)$ & $0(0 \%)$ & & $<0.01$ \\
\hline
\end{tabular}

Table 1. The proportion of different types of $H$. pylori infection in inflammation and activity.

\begin{tabular}{|c|c|c|c|c|c|}
\hline Group & Type I H. pylori infection group & Type II H. pylori infection group & Control group & & \\
\hline$n$ & 470 & 235 & 470 & $x^{2}$ & $P$ value \\
\hline Stage- 0 & $224(47.7 \%)$ & $127(54 \%)$ & $339(72.1 \%)$ & \multirow{3}{*}{67.99} & \multirow{3}{*}{$<0.01$} \\
\hline Stage-1 & $197(41.9 \%)$ & $97(41.3 \%)$ & $114(24.3 \%)$ & & \\
\hline Stage-2 & $49(10.4 \%)$ & $11(4.7 \%)$ & $17(3.6 \%)$ & & \\
\hline
\end{tabular}

Table 2. The proportion of different types of $H$. pylori infection in OLGA staging.

\section{Results}

General information. According to the 1:2 paired conditions with age and sex, 235 inpatients were enrolled in type II $H$. pylori infection group and the other two groups were 470 inpatients respectively. Among the 1175 inpatients, there were 630 males and 545 females, aging from 30 to 82 years old, with an average age of $55.9 \pm 9.4$ years. There were 470 cases in type I $H$. pylori infection group, 252 males and 218 females, with an average age of $56.1 \pm 9.1$ years; 235 cases in type II $H$. pylori infection group, 126 males and 109 females, with an average age of $56 \pm 9.5$ years; and 470 cases in control group, 215 males and 212 females, with an average age of $55.7 \pm 9.6$ years. No significant differences in sex and age among the three groups $\left(\chi^{2}=0, P=1 ; \chi^{2}=1.69, P=0.43\right.$, $P$ all $>0.05)$.

The relationship between different types of $H$. pylori infection with inflammation and activity. The proportion of different types of $H$. pylori infection in inflammation and activity was shown in Table 1. In the distribution of inflammation degree, the proportion of moderate and severe inflammation in type I $H$. pylori infection group was higher than that in type II $H$. pylori infection group and control group $\left(\chi^{2}=165.43\right.$, $P$ all $<0.01)$. In the distribution of activity degree, the proportion of mild and moderate activity in type I $H$. pylori infection group was higher than that in type II $H$. pylori infection group and control group $\left(\chi^{2}=176.22\right.$, $P$ all $<0.01)$.

Relationship between different types of $\boldsymbol{H}$. pylori infection in OLGA staging. The histopathological evaluation of gastric mucosa biopsies was performed by updated Sydney system and the staging was expressed according to OLGA classification systems. In type I $H$. pylori infection group, only two inpatients with stage-3 OLGA staging, which were included in stage-2 OLGA staging for statistics facilitate. As it depicted in Table 2, the proportion of stage-2 in type I H. pylori infection group was higher than that in type II $H$. pylori infection group and control group, the difference had statistically significant $\left(\chi^{2}=44.34, P\right.$ all $\left.<0.01\right)$. Stage- 0 in control group, type II $H$. pylori infection group and type I $H$. pylori infection group increased in turn, the difference was statistically significant $\left(\chi^{2}=67.99, P\right.$ all $\left.<0.01\right)$.

The effect of different types of $\boldsymbol{H}$. pylori infection on three items of serum gastric function. Table 3 had depicted the effect of different types of $H$. pylori infection on serum three items of serum gastric function. Compared with type II $H$. pylori infection group, in type I $H$. pylori infection group, the level of PGI, PGII and G-17 increased and the PGR decreased, the difference was statistically significant $(Z=-5.52$, $-11.97,-11.52,-10.69$, respectively; $P$ all $<0.01)$. Compared with the control group, there was no significant difference in the level of PGI, PGII, PGR $(Z=-0.54,-0.56,-0.35$; $P$ all $>0.05)$, only the level of G-17 increased $(Z=-2.31, P<0.05)$. 


\begin{tabular}{|c|c|c|c|c|c|}
\hline Group & Type I H. pylori infection group & Type II H. pylori infection group & Control group & & \\
\hline$n$ & 470 & 235 & 470 & $x^{2}$ & $P$ value \\
\hline PGI & $186.4 \pm 87.8$ & $159.8 \pm 77.3$ & $159.6 \pm 80.6$ & 35.08 & $<0.01$ \\
\hline PGII & $11.4 \pm 69.2$ & $6.36 \pm 5.1$ & $6.6 \pm 6.7$ & 166.24 & $<0.01$ \\
\hline PGR & $22.8 \pm 16.9$ & $32.9 \pm 23.3$ & $32 \pm 17.5$ & 134.21 & $<0.01$ \\
\hline G17 & $9.9 \pm 12.7$ & $6.2 \pm 10.9$ & $5.6 \pm 11.4$ & 141.19 & $<0.01$ \\
\hline
\end{tabular}

Table 3. The effect of different types of $H$. pylori infection on three items of serum gastric function.

\section{Discussion}

It is a consensus that $H$. pylori infection is the cause of chronic gastritis, and $H$. pylori can cause sustained damage to gastric mucosa. $H$. pylori is viewed as a key cause in many diseases, such as chronic gastritis with atrophy and erosion, peptic ulcer, MALT lymphoma, gastric cancer and so on. AG is considered to be the outcome of $H$. pylori infection and is associated with a high risk of gastric cancer ${ }^{15-18}$. Although eradication of $H$. pylori can reduce the risk of gastric cancer in the general population, the risk of gastric cancer do exist, which is associated with the degree of AG and IM. Histological studies showed that there is no significant change in AG and IM during the 2 years after eradication of $H$. pylori, and AG and IM are significantly improved 10 years later ${ }^{19}$.

Eradicating of $H$. pylori is not always beneficial to some diseases, because it can lead to an increase in the level of gastric acid secretion, which may increase gastroesophageal reflux and ulceration of low dose aspirin. Therefore, individualized eradication of H. pylori is the best way ${ }^{20}$. OLGA classification and serum gastric function detection are optimal for early gastric cancer screening. A surveillance program including OLGA stage and serum gastric function status may facilitate early detection of gastric cancer ${ }^{21}$. ACG Clinical Guideline have demonstrated that patients over 60 years old and unexplained dyspepsia with no early warning symptoms are recommended for non endoscopic $H$. pylori tests, and those with positive results should be treated with eradication ${ }^{22}$. H. pylori infection is one of the most important factors causing atrophy and precancerous lesions. Recently, countries around the world are actively eradicating $H$. pylori infection to reduce the incidence rate of gastric cancer. However, only few countries can eradicate $H$. pylori infection for all, indicating that individualized treatment is the feasible strategy.

$H$. pylori has a variety of diagnostic methods ${ }^{23,24}$. The most non-invasive detection methods are ${ }^{14} \mathrm{C}-\mathrm{UBT}$ and serum $H$. pylori antibody typing classification. There may be false positive and false negative results in ${ }^{14} \mathrm{C}-\mathrm{UBT}$. The combination of the two test results can accurately estimate $H$. pylori infection ${ }^{25}$. H. pylori antibody typing classification is mainly based on the expression of virulence protein CagA, VacA, Urease A and Urease B. CagA of $H$. pylori can accelerate the progress of gastric cancer and AG, in which tyrosine phosphorylated EPIYA modules play an important role in active gastritis, associating with the progression of diffuse-type of gastric cancer, especially in women ${ }^{26}$. The meta-analysis has summarized 33 studies and points that H. pylori CagA genotypes are associated with increased risk of AG, IM, and gastric cancer, especially IM and gastric cancer ${ }^{27}$. CagA positive $H$. pylori can downregulation of autophagy in the gastric mucosa and induce the increase of inflammatory factors and increase the degree of gastritis ${ }^{28}$. Through different types of $H$. pylori infection and histopathological characteristics analysis, it found that the degree of inflammation and activity caused by type I H. pylori was significantly higher than that caused by type II H. pylori. According to OLGA classification systems, histopathological evaluation results showed that type I H. pylori tended to a higher staging levels. Thus, it is speculated that $H$. pylori infection and its pathogenicity are closely related to the specific genes and proteins. The results suggested that different types of $H$. pylori infection had different characteristics, as well as explained that most of the patients with $H$. pylori infection did not have any symptoms.

In the gastric epithelial cells, CagA is transmitted through a unique type IV secretory system to the initial immune response, followed by a series of responses to recruit immune cells to form chronic gastritis, and serum CagA and VacA virulence proteins increase the risk of non cardia cancer, while the risk of low virulence protein of $H$. pylori is lower ${ }^{29,30}$. Serological detection of pepsinogen PG I, PGII, PGR and G-17, the epidemiological markers for gastric cancer risk investigation, has provided valuable information for the status of gastric mucosa ${ }^{31}$. This study showed that type I $H$. pylori infection could increase the level of serum PGI, PGII and G-17, possibly because the inflammatory mechanism. The study of $H$. pylori virulence factors were focused on the gene level, depicting that $\operatorname{CagA}$ and $\operatorname{VacA}$ gene positive samples have strong correlation with AG and IM, and can better predict $\mathrm{AG}$ and $\mathrm{IM}^{32,33}$. Some studies have found that the invasion ability of $H$. pylori infection is associated with the middle region of the $v a c A$ gene ${ }^{34,35}$. This result suggested that different types of $H$. pylori infection might be related to the size of the invasiveness. According to the different secretory sites of PGI and PGII in the stomach, it is speculated that type I $H$. pylori infection could increase the secretion of serum PG in the whole stomach and duodenum region. The infection of type I $H$. pylori infection might accelerate the progress of atrophic gastritis due to the increase the invasion of gastric mucosa and the level of serum gastric function through unique inflammatory mechanisms.

In conclusion, different types of $H$. pylori had different characteristics. Through the analysis of histopathological characteristics and serum gastric function, it is confirmed that type I $H$. pylori infection played a key role in worsening the progress of atrophic gastritis, while the role of type II H. pylori infection is relatively weak. Thus, the clinical application of $H$. pylori eradication should be differentiated based on the $H$. pylori antibody typing classification and individualizing eradication of $H$. pylori can benefit more people. 
Received: 25 October 2020; Accepted: 5 February 2021

Published online: 18 February 2021

\section{References}

1. Witkowska, M., Smolewski, P. Helicobacter pylori infection, chronic inflammation, and genomic transformations in gastric MALT lymphoma. Mediators Inflamm. 523170, 1-8 (2013).

2. Miyake, K. et al. Implications of corpus gastritis, atrophy and cyclooxygenase in the development of gastric erosions after curing Helicobacter pylori infection. Dig. Liver Dis. 37(6), 394-401 (2005).

3. Muzaheed. Helicobacter pylori oncogenicity: Mechanism, prevention, and risk factors. Sci. World J. 3018326, 1-10 (2020).

4. Timothy, L. Cover: Helicobacter pylori diversity and gastric cancer risk. mBio 7(1), e01869-15 (2016).

5. Zhang, B. B. et al. Association between vacA genotypes and the risk of duodenal ulcer: a meta-analysis. Mol. Biol. Rep. 41(11), 7241-7254 (2014).

6. Bakhti, S. Z. et al. Unique constellations of five polymorphic sites of Helicobacter pylori vac A and cag A status associated with risk of gastric cancer. Infect. Genet. Evol. 79, 104167 (2020).

7. Muhsen, K. et al. Sero-prevalence of Helicobacter pylori CagA immunoglobulin G antibody, serum pepsinogens and haemoglobin levels in adults. Sci. Rep. 8(1), 17616 (2018).

8. Hwang, Y. J. et al. Reversibility of atrophic gastritis and intestinal metaplasia after Helicobacter pylori eradication-A prospective study for up to 10 years[J]. Aliment Pharmacol. Ther. 47(3), 380-390 (2018).

9. Sjomina, O. et al. Pepsinogen test for the evaluation of precancerous changes in gastric mucosa: A population-based study. J. Gastrointestin. Liver Dis. 27(1), 11-17 (2018).

10. Su, W. et al. Low PG I/II ratio as a marker of atrophic gastritis: Association with nutritional and metabolic status in healthy people. Medicine (Baltimore) 97(20), e10820 (2018).

11. Chen, X. Z. et al. Gastric cancer screening by combined determination of serum Helicobacter pylori antibody and pepsinogen concentrations: ABC method for gastric cancer screening. Chin. Med. J. (Engl.) 131(10), 1232-1239 (2018).

12. Shafaghi, A. et al. Serum gastrin and the pepsinogen I/II ratio as markers for diagnosis of premalignant gastric lesions. Asian Pac. J. Cancer Prev. 14(6), 3931-3936 (2013).

13. Annibale, B., Esposito, G. \& Lahner, E. A current clinical overview of atrophic gastritis. Exp. Rev. Gastroenterol. Hepatol. 14(2), 93-102 (2020).

14. Chinese Society Of Gastroenterology. Chinese consensus on chronic gastritis (Shanghai, 2017). Chin. J. Digest. 37(11), 721-738 (2017).

15. Lahner, E. et al. Immunoproteomics of Helicobacter pylori infection in patients with atrophic body gastritis, a predisposing condition for gastric cancer. Int. J. Med. Microbiol. 301, 125-132 (2011).

16. Liu, W. Z. et al. Fifth Chinese National Consensus Report on the management of Helicobacter pylori infection. Helicobacter 23(2), e12475 (2018).

17. Zagari, R. M. et al. Guidelines for the management of Helicobacter pylori infection in Italy: The III Working Group Consensus Report 2015. Dig. Liver Dis. 47(11), 903-912 (2015).

18. Fallone, C. A. et al. The Toronto Consensus for the treatment of Helicobacter pylori infection in adults. Gastroenterology 15(1), 51-69 (2016).

19. Kodama, M. et al. Ten-year prospective follow-up of histological changes at five points on the gastric mucosa as recommended by the updated Sydney system after Helicobacter pylori eradication. J. Gastroenterol. 47, 394-403 (2012).

20. Sugano, K. et al. Kyoto global consensus report on Helicobacter pylori gastritis. Gut 64(9), 1353-1367 (2015).

21. Zhou, Y. et al. Operative link on gastritis assessment stage is an appropriate predictor of early gastric cancer. World J. Gastroenterol. 22(13), 3670-3678 (2016).

22. Chey, W. D. et al. ACG clinical guideline: Treatment of Helicobacter pylori infection. Am. J. Gastroenterol. 112(2), 212-239 (2017).

23. Elfant, A. B., Howden, C. W. \& Stollman, N. Contemporary diagnostic strategies for the detection of Helicobacter pylori infection. Gastroenterol. Hepatol. (NY) 8(11 Suppl 7), 1-16 (2012).

24. Skrebinska, S. et al. Accuracy of two plasma antibody tests and faecal antigen test for non-invasive detection of $H$. pylori in middleaged Caucasian general population sample. Scand. J. Gastroenterol. 53(7), 777-783 (2018).

25. Shafaie, E. et al. Multiplex serology of Helicobacter pylori antigens in detection of current infection and atrophic gastritis-A simple and cost-efficient method. Microb. Pathog. 119, 137-144 (2018).

26. Wada, Y. et al. Relationship between Helicobacter pylori tyrosine-phosphorylated CagA-related markers and the development of diffuse-type gastric cancers: A case-control study. Digestion 82(1), 10-17 (2010).

27. Abdi, E. et al. Helicobacter pylori vacuolating cytotoxin genotypes and preneoplastic lesions or gastric cancer risk: A meta-analysis. J. Gastroenterol. Hepatol. 31(4), 734-744 (2016).

28. Li, N. et al. Helicobacter pylori CagA protein negatively regulates autophagy and promotes inflammatory response via c-Met-PI3K/ Akt-mTOR signaling pathway. Front. Cell Infect. Microbiol. 7, 417 (2017).

29. Gall, A. et al. TIFA signaling in gastric epithelial cells initiates the cag type 4 secretion system-dependent innate immune response to Helicobacter pylori infection. Mbio 8(4), e01168-e1217 (2017).

30. de Larrea-Baz, N. F. et al. Helicobacter pylori serological biomarkers of gastric cancer risk in the MCC-Spain case-control study. Cancer Epidemiol. 50(Pt A), 76-84 (2017).

31. Yuan, L. et al. Type I and type II Helicobacter pylori infection status and their impact on gastrin and pepsinogen level in a gastric cancer prevalent area. World J. Gastroenterol. 26(25), 3673-3685 (2020).

32. Pan, K. F. et al. Helicobacter pylori antibody responses and evolution of precancerous gastric lesions in a Chinese population. Int. J. Cancer 134(9), 2118-2125 (2014).

33. Filomena, A. et al. Performance of a multiplex serological Helicobacter pylori assay on a novel microfluidic assay platform. Proteomes 5(4), 24 (2017).

34. Zhang, X. et al. Analysis of the relationship between invasive capability of Helicobacter pylori and gastroduodenal diseases. J. Med. Microbiol. 64, 498-506 (2015).

35. Jin, H.F., Dai, J.F., Meng, L.N., Lu, B. Curcuma wenyujin Y. H. Chen et C. Ling n-Butyl alcohol extract inhibits AGS cell Helicobacter pylori CagA + VacA + promoted invasiveness by down-regulating caudal type homeobox transcription factor and claudin-2 expression. Chin. J. Integr. Med. 26(2), 122-129 (2017).

\section{Author contributions}

W.L. and J.T. are the experimental designers and executors of this study; W.H., W.K., and Y.F. participate in the experimental design and analysis of the experimental results; J.S. and F.G. are the designers and responsible persons of the project, guiding the experimental design, data analysis, thesis writing and revision. 


\section{Funding}

Natural Science Foundation of Xinjiang Uygur Autonomous Region (2019D01C110).

\section{Competing interests}

The authors declare no competing interests.

\section{Additional information}

Correspondence and requests for materials should be addressed to J.S. or F.G.

Reprints and permissions information is available at www.nature.com/reprints.

Publisher's note Springer Nature remains neutral with regard to jurisdictional claims in published maps and institutional affiliations.

(c) (i) Open Access This article is licensed under a Creative Commons Attribution 4.0 International License, which permits use, sharing, adaptation, distribution and reproduction in any medium or format, as long as you give appropriate credit to the original author(s) and the source, provide a link to the Creative Commons licence, and indicate if changes were made. The images or other third party material in this article are included in the article's Creative Commons licence, unless indicated otherwise in a credit line to the material. If material is not included in the article's Creative Commons licence and your intended use is not permitted by statutory regulation or exceeds the permitted use, you will need to obtain permission directly from the copyright holder. To view a copy of this licence, visit http://creativecommons.org/licenses/by/4.0/.

(C) The Author(s) 2021 